\title{
A dark state of Chern bands: Designing flat bands with higher Chern number
}

\author{
Mateusz Łącki ${ }^{1}$, Jakub Zakrzewski ${ }^{1,2}$, Nathan Goldman ${ }^{3^{*}}$ \\ 1 Institute of Theoretical Physics, Jagiellonian University in Krakow, Łojasiewicza 11, \\ 30-348 Kraków, Poland \\ 2 Mark Kac Complex Systems Research Center, Jagiellonian University in Krakow, \\ Łojasiewicza 11, 30-348 Kraków, Poland \\ 3 CENOLI, Université Libre de Bruxelles, CP 231, Campus Plaine, B-1050 Brussels, Belgium \\ * Nathan Goldman ngoldman@ulb.ac.be
}

September 23, 2020

\begin{abstract}
We introduce a scheme by which flat bands with higher Chern number $|C|>1$ can be designed in ultracold gases through a coherent manipulation of Bloch bands. Inspired by quantum-optics methods, our approach consists in creating a "dark Bloch band", by coupling a set of source bands through resonant processes. The Chern number of the dark band is found to follow a simple sum rule in terms of the Chern numbers of the source bands. We illustrate our method based on a $\Lambda$ system, formed of the bands of the Harper-Hofstadter model, which leads to a very flat Chern band with $C=2$. We explore a realistic sequence to load atoms into the dark Chern band, as well as a probing scheme based on Hall drift measurements. Dark Chern bands offer a practical platform where exotic fractional quantum Hall states could be realized in ultracold gases.
\end{abstract}

\section{Contents}

1 Introduction 2

2 Dark state of a $\Lambda$ system 3

3 The $\Lambda$ system of topological Bloch states and the sum rule 4

4 The multi-species configuration 5

5 Higher Chern number from centre-of-mass responses

6 Conclusion $\quad 9$

A Dark state formula 9

B Rotating Wave Approximation $\quad$ 10] 
C Dark state stability and the flatness of bands 11 C.1 Dark state stability 11

C.2 Band flatness and Rabi frequencies variation in the Harper-Hofstadter model 13

C.3 Band flatness - flatness factor $f$ 14

C.4 Rabi frequencies total variation - factor $g$

D Numerical evaluation of Chern numbers and the sum-rule validation 16

References

\section{Introduction}

Designing Bloch bands with topological properties has become a central theme in the context of quantum-engineered systems [1-3]. In ultracold atoms, important efforts dedicated to the realization of emblematic topological lattice models [3, 4], such as the Haldane [5, 6] and the Harper-Hofstadter models $7-10$, have recently led to the observation of a variety of topological phenomena, including quantized transport [9, 11, 14], chiral edge motion [14 18], vortex dynamics upon a quench [6, 19], and quantized circular dichroism [20. Of particular interest is the possibility of engineering flat bands with non-trivial Chern number, which could allow for the creation of strongly-correlated topological states reminiscent of fractional quantum Hall states 21 . While flat bands with Chern number $|C|=1$ are reminiscent of the conventional Landau levels in the continuum [22, flat bands with higher Chern number $|C|>1$ can lead to exotic strongly-correlated states that are specific to lattice systems [23 29]. However, such flat-band models remain unsuitable for cold-atom experiments, as they rely on the design of complicated multi-layered lattices or complex long-range hopping processes [21, 24, 25, 30, 35].

In this work, we introduce a practical approach by which flat bands with large Chern number can be designed in ultracold gases, using realistic optical-lattice geometries. Inspired by the concept of "dark state" in quantum optics [36, 37], our scheme relies on coupling a set of Bloch bands coherently in view of forming a non-degenerate "dark band". In the simplest $\Lambda$ scheme scenario, which involves three Bloch bands [Fig. 1], the Chern number of the engineered dark band is found to be dictated by a sum rule, $C_{D}=C_{1}+C_{2}-C_{3}$, where $C_{1,2,3}$ designate the Chern numbers associated with the three bare Bloch bands. Moreover, the flatness of the dark band is shown to be directly inherited from the bare bands. Altogether, this strategy offers a practical method for designing flat bands with controllable topological invariants in a wide range of cold-atom settings.

In the following, we discuss in detail the applicability and validity of this general approach, in view of realizing flat bands with higher Chern number in available cold-atom setups. In particular, we describe a realistic method by which atoms can be loaded into the topological dark band. Besides, we present clear signatures of the prepared state based on Hall drift measurements $[9,38,39$. While our results directly apply to cold-atom settings, our findings are general and could be relevant to light-induced topological phases in the solid state 40,41. 

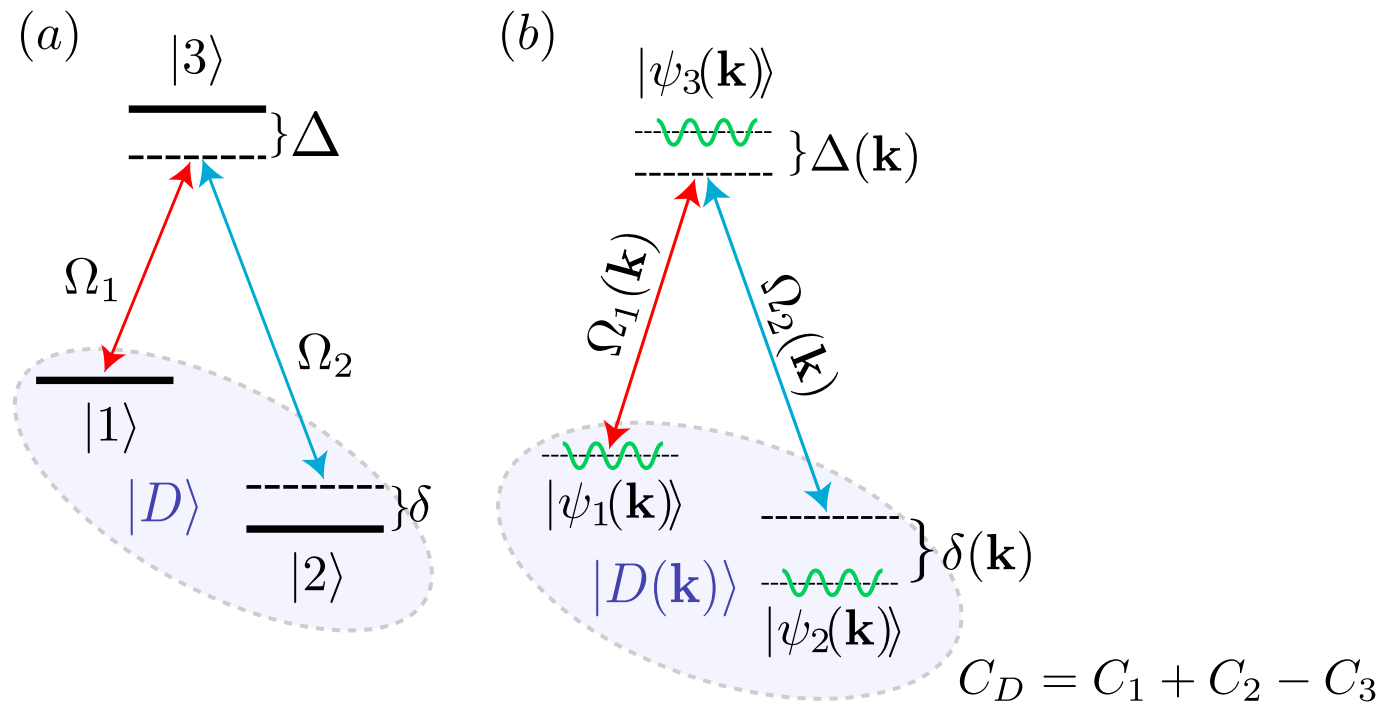

Figure 1: (a): The $\Lambda$ scheme involving three discrete levels. The Rabi couplings $\Omega_{1,2}$ and frequency detunings $\Delta$ and $\delta$ are indicated; see Eq. (1). A stable dark state $|D\rangle$ is created when $\delta \approx 0$; see Eq. (2). (b) The $\Lambda$ scheme involving three Bloch bands $E_{1,2,3}(\mathbf{k})$. The detunings and Rabi frequencies depend on the quasimomentum $\mathbf{k}$ of the Bloch states. The Chern number of the resulting "dark band" $C_{D}$ follows a simple sum rule in terms of the Chern numbers $C_{1,2,3}$ of the bare bands.

\section{Dark state of a $\Lambda$ system}

The dark-band scheme proposed and analyzed in this work is based on a simple three-band configuration, which is reminiscent of the well-known $\Lambda$ scheme of quantum optics [37]. As depicted in Fig. 1(a), a generic $\Lambda$ scheme involves three quantum states $|1\rangle,|2\rangle,|3\rangle$, and is described by the Hamiltonian

$$
\hat{H}_{\Lambda}=\hbar\left(\begin{array}{ccc}
0 & 0 & \Omega_{1}^{*} / 2 \\
0 & \delta & \Omega_{2}^{*} / 2 \\
\Omega_{1} / 2 & \Omega_{2} / 2 & -\Delta
\end{array}\right)
$$

For $\delta / \Omega_{1,2} \rightarrow 0$ and arbitrary $\Delta$, the dark state (DS),

$$
|D\rangle=\frac{1}{2 \bar{\Omega}}\left(\Omega_{1}|2\rangle-\Omega_{2}|1\rangle\right), \quad \bar{\Omega}=\frac{1}{2} \sqrt{\left|\Omega_{1}\right|^{2}+\left|\Omega_{2}\right|^{2}},
$$

is an eigenstate of $\hat{H}_{\Lambda}$ corresponding to the eigenvalue $E_{0}=0$. The Hamiltonian $\hat{H}_{\Lambda}$ possesses two other "bright" eigenstates, with energies $E_{ \pm}=\frac{\hbar}{2}\left(-\Delta \pm \sqrt{\Delta^{2}+4 \overline{\Omega^{2}}}\right)$. In contrast with the dark state in Eq. (2), which forms a robust superposition of the two low-lying states $(|1\rangle$ and $|2\rangle)$ only, the bright states involve all components [37]; see Appendix A for a more detailed review.

We now apply this dark-state notion, which has been widely exploited in quantum optics $[4252$ and atomic physics [53 59], to the case of three coherently coupled Bloch bands $E_{1,2,3}(\mathbf{k})$, where $\mathbf{k}$ denotes quasi-momentum. 


\section{The $\Lambda$ system of topological Bloch states and the sum rule}

For the sake of concreteness, we consider the Bloch bands of the emblematic Harper-Hofstadter $(\mathrm{HH})$ model [60], which can be engineered in optical-lattice experiments [7 $[10]$. The corresponding Hamiltonian reads

$$
\hat{H}_{\mathrm{HH}}=-J \sum_{\mathbf{n}=(n, m)} \hat{a}_{\mathbf{n}}^{\dagger} \hat{a}_{\mathbf{n}+\mathbf{1}_{\mathbf{y}}}+\hat{a}_{\mathbf{n}}^{\dagger} \hat{a}_{\mathbf{n}+\mathbf{1}_{\mathbf{x}}} e^{i 2 \pi m \phi}+\text { h.c. }
$$

where $\mathbf{n}=(n, m)$ enumerates lattice sites on a $2 \mathrm{D}$ square lattice with lattice constant $a, J$ is the hopping amplitude, and where $a_{\mathbf{n}} / a_{\mathbf{n}}^{\dagger}$ annihilate/create a particle at site $\mathbf{n}$. The complex phase factor, which accompanies hopping along the $x$ direction and generates a uniform magnetic flux $2 \pi \phi$ per plaquette, can be tuned in experiments $[7-10]$.

Considering a flux of the form $\phi=1 / q$, with $q$ an odd integer, and applying periodic boundary conditions $(\mathrm{PBC})$, the spectrum displays $q$ non-degenerate Bloch bands $\varepsilon_{\nu}(\mathbf{k})$, where $\nu=1, \ldots, q$. We define the first Brillouin zone (BZ) as $\mathbf{k} \in[0,2 \pi / a q) \times[0,2 \pi / a)$. Each band is associated with a topological Chern number 61

$$
C_{\nu}=\frac{1}{2 \pi} \int_{B Z} \mathcal{F} d^{2} k, \mathcal{F}=2 \operatorname{Im}\left\langle\partial_{k_{y}} \psi_{\nu}(\mathbf{k}) \mid \partial_{k_{x}} \psi_{\nu}(\mathbf{k})\right\rangle
$$

where $\psi_{\nu}(\mathbf{k})$ denotes an eigenstate in the $\nu$ th band. For $q$ a generic odd integer, the Chern number of the central band $[\nu=(q+1) / 2]$ reads $C_{\nu}=(-q+1)$, while $C_{\nu}=1$ for all the other bands. Hence, except from the central band, the bands of the $\mathrm{HH}$ model are reminiscent of Landau levels (LL). In particular, these LL-like bands become flat in the limit $q \gg 1$.

We now discuss how a coherent coupling of these LL-like bands allow for the generation of flat bands with higher Chern number $|C|>1$ within the HH model. This is achieved by building a $\Lambda$ system [Eq. (1)] from three selected bands $E_{1,2,3}(\mathbf{k})$ of the HH spectrum $\left\{\varepsilon_{\nu}\right\}$; we will denote the mean energy of each band as $\bar{E}_{1,2,3}$. In order to couple these bands quasiresonantly, we consider a two-frequency drive with frequencies set to $\hbar \omega_{1,2}=\bar{E}_{1,2}-\bar{E}_{3}$; see Fig. 1(b). Assuming that the coupling field has a spatial periodicity that is compatible with the (magnetic) unit cell, the system forms a collection of decoupled $\Lambda$ systems, one at each quasi-momentum k. Applying the rotating wave approximation (RWA) [37], as detailed in Appendix B, and neglecting the other bands, this setting is indeed well described by Eq. (1), with momentum-dependent Rabi frequencies and detunings.

Introducing the corresponding dark states, $|D(\mathbf{k})\rangle$, we numerically evaluate the Chern number of the dark band, and we find the following sum rule:

$$
C_{D}=C_{1}+C_{2}-C_{3}
$$

where $C_{1,2,3}$ denote the Chern numbers of the selected bands $E_{1,2,3}$. Our broad numerical investigations (Appendix $\mathrm{D}$ ) indicate that this simple sum rule is independent of: (i) the flux $\phi=1 / q$, (ii) the operator associated with the coupling field, and (iii) the chosen bands in the $\mathrm{HH}$ spectrum. Altogether, this provides a unique way to produce bands with higher Chern number. We point out that the sum rule (5) differs from that recently found in multilayer lattice systems 62 .

The formula (5) is valid whenever an effective $\Lambda$ (three-band) configuration is achieved and the dark-state band is well separated from the two bright-state bands. This imposes a 
series of constraints on the chosen bands and system parameters, as we now summarize; see also Appendix C for further details.

First, the finite bandwidth of the bare bands $E_{1,2,3}(\mathbf{k})$ produces detunings $\delta(\mathbf{k})$ and $\Delta(\mathbf{k})$ in Eq. (1), which affects the flatness of the dark band and reduces the gap to the bright bands; see Appendix C.1. This effect can be limited by noting that the width of the $\mathrm{HH}$ bands decreases exponentially with $q$ (except for the central band); see Appendix C.2.

Then, the Rabi frequencies $\Omega_{1,2}(\mathbf{k})$ should satisfy the inequalities $\delta(\mathbf{k}) \ll \Omega_{1,2}(\mathbf{k}) \ll$ $\left|E_{ \pm}(\mathbf{k})\right|$ to allow for a good separation of the dark band and optimize its flatness; this condition can already be reached for moderate $q \sim 7-10$. Besides, one should avoid pathological zeros $\bar{\Omega}(\mathbf{k})=0$, which would invalidate the dark state construction; see Appendix C.4.

Furthermore, for large $q \gg 1$, the $\mathrm{HH}$ spectrum forms a ladder of quasi-equally spaced Landau levels, which indicates that only special choices of bare bands $E_{1,2,3}$ can lead to a genuine $\Lambda$ configuration. In addition, the RWA is only valid when the resonant frequencies $\omega_{1,2}$ (and their differences $\Delta \omega$ ) are much larger than all other frequency scales, which also sets an important constraint on the chosen bands.

Finally, the unique bare band with $C_{\nu} \neq 1$ is the central band $[\nu=(q+1) / 2]$, for which the bandwidth to bandgap ratio is $O(1)$. This unfortunately rules out involving this band in our construction, which eventually implies the disappointing result $C_{D}=1$ [Eq. (5)].

In order to overcome these limitations and constraints, we slightly generalize our scheme by introducing different atomic species (i.e. "spins"), as we now describe in the next section.

\section{The multi-species configuration}

We propose to generate a flat and non-degenerate dark band with $C_{D}=2$, by constructing a $\Lambda$ system made of two HH bands $\left[C_{1,2}=1\right]$ and a trivial band $\left[C_{3}=0\right]$. The latter is provided by the lowest band of a square lattice without flux [Eq. (3) with $\phi=0$, denoted $\hat{H}_{0}$ ], whose unit cell area $A_{\text {cell }}=q a \times a$ ensures a common BZ with $\hat{H}_{\mathrm{HH}}$; the corresponding sites are located at $\tilde{\mathbf{n}}=(q n, m)$. This setting could be realized using different atomic (internal) states trapped in state-dependent potentials [64 66]; see Refs. 67, 67, 68, for schemes realizing state-dependent synthetic flux. In order to guarantee the validity of the RWA, we henceforth consider that each of the three selected bands $E_{1,2,3}$ is populated by a specific internal state $\sigma=\{1,2,3\}$. The $\Lambda$ coupling between the three bands is then performed by properly coupling the internal states with microwave fields. We note that using only two internal states could also be envisaged in practice.

Upon the RWA, the Hamiltonian of this setting reads

$$
\hat{H}_{\Lambda}=\sum_{\sigma=1}^{3}\left(\hat{H}_{\sigma}+\delta_{\sigma} \hat{P}_{\sigma}\right)+\frac{1}{2} \sum_{s=1,2} A_{s} \sum_{\tilde{\mathbf{n}}=(q n, m)} \hat{a}_{\tilde{\mathbf{n}}, s}^{\dagger} \hat{a}_{\tilde{\mathbf{n}}, 3}+\text { h.c. }
$$

where $a_{\tilde{\mathbf{n}}, \sigma}^{\dagger}$ creates an atom at site $\tilde{\mathbf{n}}$ in internal state $\sigma ; \hat{H}_{1,2}=\hat{H}_{H H} \otimes \hat{P}_{1,2}$ and $\hat{H}_{3}=\hat{H}_{0} \otimes \hat{P}_{3}$, where $\hat{P}_{\sigma}$ projects onto the $\sigma$ component; $A_{1,2}$ denote the amplitudes of the coupling fields, and the detunings $\delta_{\sigma}$ are controlled by tuning the driving frequencies out of resonance. In particular, these detunings can be chosen such that two bands of the HH spectrum (populated by states $\sigma=1,2$ ) become degenerate with the trivial band (populated by $\sigma=3$ ) in the decoupled limit $A_{1,2}=0$; see Figs. 2(a)-(b). Upon activating the coupling $\left(A_{1,2} \neq 0\right)$, these 

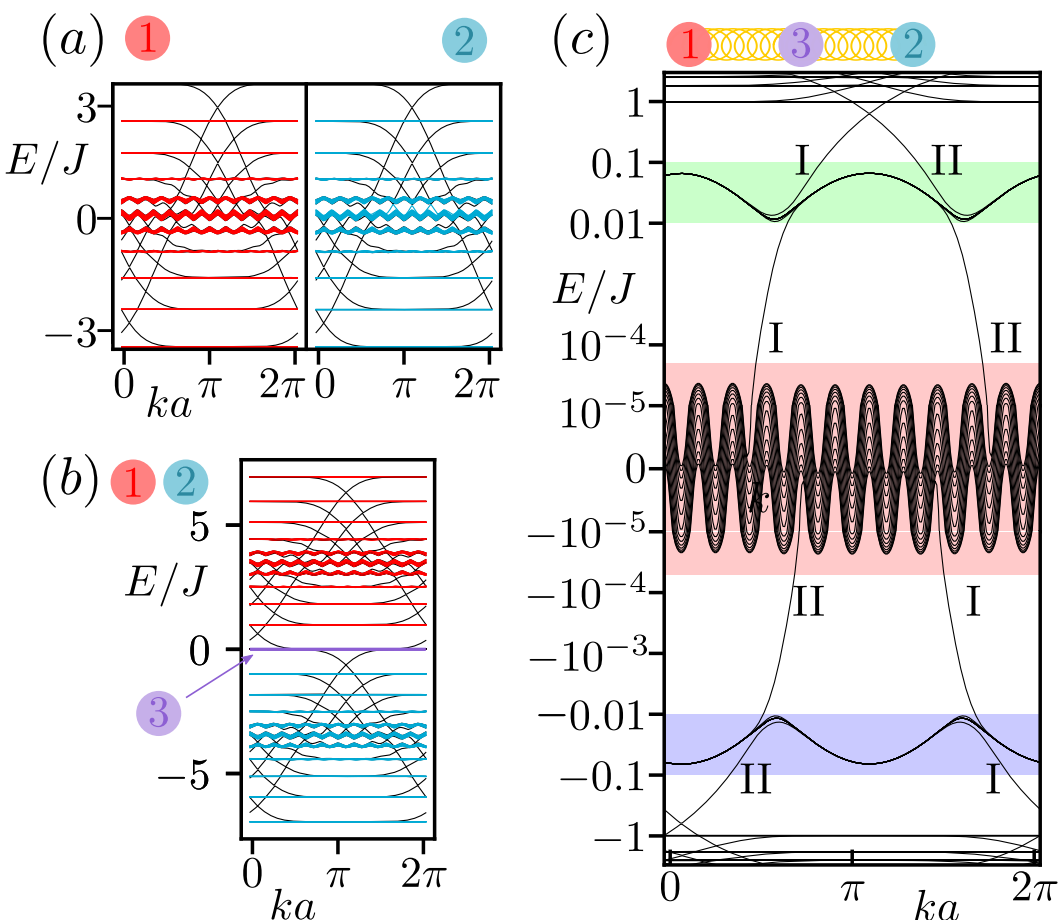

Figure 2: Designing a $\Lambda$ system from two Hofstadter bands and a trivial band. (a) Hofstadter bands associated with two uncoupled atomic internal states $(\sigma=1,2)$, for a flux $\phi=1 / 11$. The black dispersions correspond to edge-state branches [63]; the system is diagonalized on a cylindrical geometry. (b) Adjusting the detunings $\delta_{\sigma}$ in $\bar{H}_{\Lambda}$ such that two Hofstadter bands (populated by states $\sigma=1,2$ ) become degenerate with a flat trivial band (populated by $\sigma=3$ ) in the uncoupled limit $A_{1,2}=0$. (c) Activating the coupling $\left(A_{1,2} \neq 0, \max \left|\Omega_{1,2}(\mathbf{k})\right|=J / 5\right)$ splits the three overlapping bands into a flat dark band at zero energy (red shaded), and two bright bands (green/purple shaded). The flatness of the dark band is emphasized by a logarithmic scale. The edge-state branches, associated with the two edges (I,II) of the cylinder, indicate that the Chern number of the dark band is $C_{D}=2$. 
three bands split into the dark band and the two bright bands shown in Fig. 2(c). While the bright bands acquire a small dispersion, due to the $\mathbf{k}$-dependence of the Rabi frequencies $\Omega_{1,2}(\mathbf{k})$, the dark band at zero energy remains almost perfectly flat; see Appendix C.4.

One verifies that the Chern number of the dark band is $C_{D}=2$, as dictated by the sum rule (5); this is readily obtained by analyzing the edge-state branches of opposite chirality that enter and leave the dark band in Fig. 2(c); see Ref. [63]. One also finds that the Chern number of the bright bands are zero, such that the total Chern number of the three coupled bands is indeed conserved. We have verified that these results are generic, in the sense that they do not depend on the specific form of the coupling operator; further details on our numerical explorations are provided in Appendix D.

\section{$5 \quad$ Higher Chern number from centre-of-mass responses}

The Chern number $C_{D}$ of the constructed dark band could be measured through different probes in ultracold atoms, such as center-of-mass responses [9], edge-state spectroscopy [69,70], and circular dichroism [20]. Here, we validate our approach by simulating the center-of-mass displacement of an atomic cloud, loaded in the dark band, and perturbed by a linear potential gradient $[9,38,39]$.

As a first step, we numerically simulate the following protocol [38]: We initially confine the system, using sharp rectangular walls; we generate a dark band [as illustrated in Fig. 2(c)] in this geometry and completely fill it with non-interacting fermions; we remove the confining walls, and act on the particles with a weak linear potential gradient aligned along the $y$ direction, $\hat{F}=F a \sum_{s=1,2} \sum_{\mathbf{n}=(n, m)} m \hat{n}_{\mathbf{n}, s}$; finally, we calculate the time evolution of the particle density $\rho(x, y)$, and evaluate the center-of-mass displacement $\Delta x(t)$. The latter observable is related to the Chern number of the populated band $\left(C_{D}\right)$ through the relation $9,38,39$

$$
\Delta x(t)=\frac{q a^{2} F}{h} C_{D} t, \quad \text { for a flux } \phi=1 / q .
$$

We show the simulated time-evolved density in Figs. 3(a)-(b), which demonstrate a clear transverse drift of the cloud (along the $x$ direction). We note that the density modulation along the $x$ direction reflects the coupling to the "trivial" lattice, of area $A_{\text {cell }}=q a \times a$, which supports the component $\sigma=3$ (which is absent in the dark state). In Fig. 3(a), the applied force is weak compared to the gap $\Delta_{\mathrm{db}}$ separating the dark band from the bright bands, $F a \ll \Delta_{\mathrm{db}}$, such that the particles' motion adiabatically follows the dark band. In this linear-response regime, Eq. (7) is applicable [38,39], and we extract an "experimental" value for the Chern number of the dark band $C_{D}^{\exp }=1.99$ from the center-of-mass drift [Fig. 3(f)]. In contrast, the force is strong $F a>\Delta_{\mathrm{db}}$ in the case depicted in Fig. 3(b), which leads to a dynamical repopulation of the bright states. Importantly, these undesired excitations are clearly identified by narrow stripes of highly populated sites, separated by qa along the $x$ direction. For completeness, we also calculated the time-evolved density upon populating a bare Hofstadter band (with $C=1$ ) instead of the dark band: the resulting Fig. 3(c), when compared with Fig. 3(a), reveals that the center-of-mass velocity indeed differs by the predicted factor of two; see also Fig. $3(\mathrm{f})$.

Finally, we explore a realistic sequence for loading atoms into the designed dark band of Fig. 2. We start the sequence by completely filling the lowest Hofstadter band with a single species $(\sigma=1)$, in the absence of coupling to the other species $(\sigma=2,3)$. This initial state is a Chern insulator with Chern number $C_{1}=1$. Considering the $\Lambda$ scheme of Eq. (6), one then 

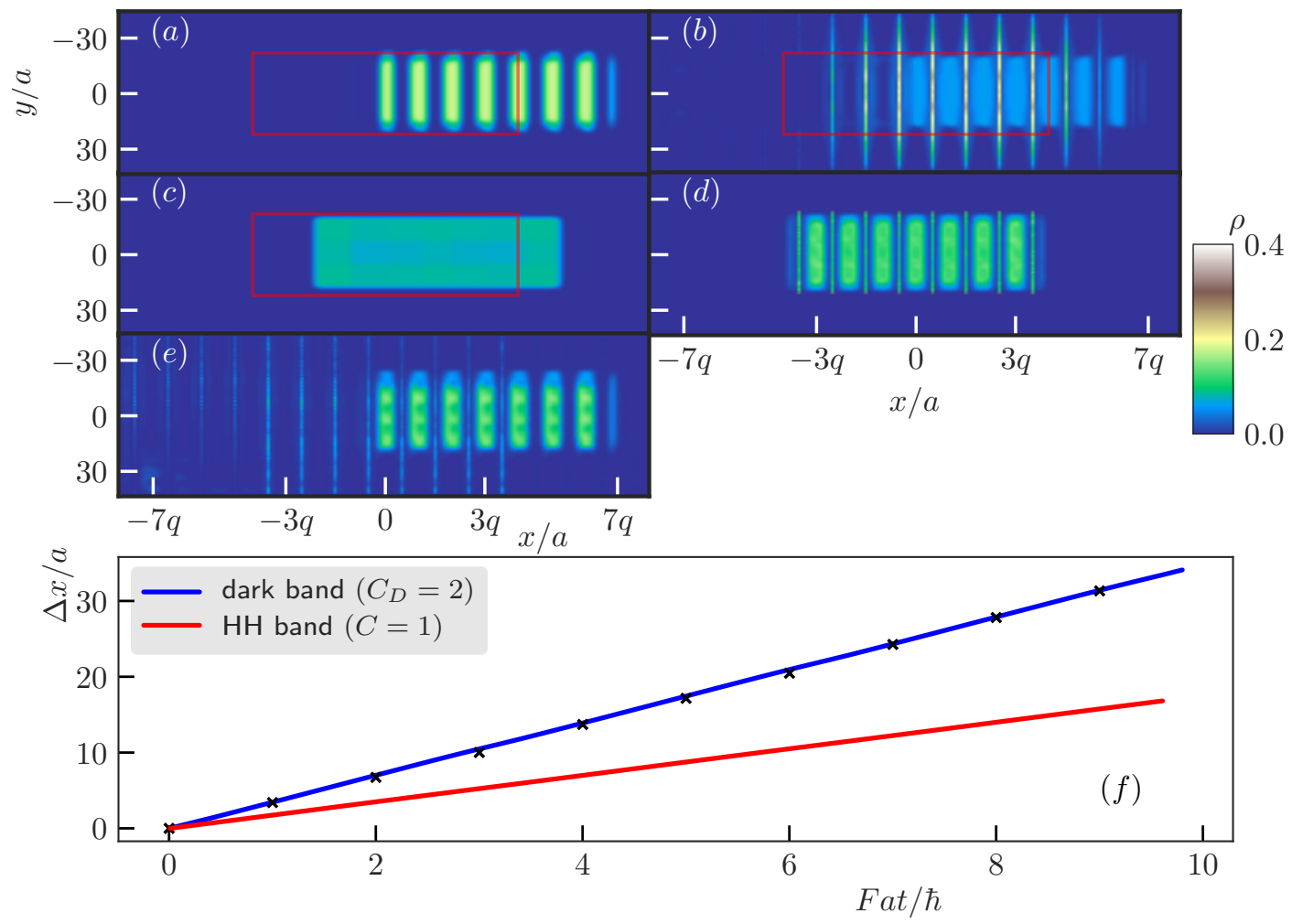

Figure 3: Hall drift in the dark band. (a) Time-evolved density $\rho(x, y)$ of non-interacting fermions, initially confined within a small region (red rectangle) and populating the dark band, and then exposed to a linear gradient along $y$ upon release. Here the force is weak compared to the gap to the bright states, $F a=5 \cdot 10^{-4} \mathrm{~J} \ll \Delta_{\mathrm{db}}=5 \cdot 10^{-2} \mathrm{~J}$, allowing for an accurate measurement of the Chern number $C_{D}^{\exp }=1.99$ through the center-of-mass drift [panel (f) and Eq. (7)]. The evolution duration is $t=15(\hbar / F a)$ and the flux $\phi=1 / 11$. (b) Same protocol but using a larger force, $F=5 \cdot 10^{-3} \mathrm{~J} / \mathrm{a}$, leading to a substantial repopulation of the bright bands over long times (thin stripes in the density). (c) Same but populating a regular Hofstadter band (with $C=1$ ) instead of the dark band. (d) Density at the end of the loading sequence (main text). (e) Time-evolved density of the realistically prepared state in (d), when exposed to the linear gradient along $y$. (f) Center of mass drift for the manually-loaded dark band (blue line), the bare Hofstadter band (red line), and for the realistically-prepared state (black crosses); in the latter case, residual bright-state contributions were filtered out (main text).

progressively activates the couplings $\left(A_{1,2} \neq 0\right)$ so as to adiabatically transfer the atoms from the bare Hofstadter band to the dark band with $C_{D}=2$. Because of the change in the Chern number, non-adiabatic effects are unavoidable, so that this final state cannot be reached with $100 \%$ fidelity. However, a dominating population in the target dark band can be obtained upon slowly ramping up the coupling $A_{1}$ to its final value 9,71 . We perform a simulation of this sequence, by calculating the time evolution of single-particle states according to the time-dependent Hamiltonian $H_{\Lambda}\left[A_{1}(t)\right]$, where $A_{1}(t)=0 \rightarrow A_{1}$. The density of the final state 
is shown in Fig. 3(d), where the thin vertical stripes separated by $q a$ again highlight the residual population in the bright states. In our simulated setting, we find a $10 \%$ fraction in the component $\sigma=3$ for a linear ramp of duration $T \approx 10^{6}(\hbar / J)$. We note that this undesired population can be significantly reduced by optimizing the ramp function, or by increasing the system size and ramp duration; other state-preparation protocols, based on optimal-control theory 72 74 and nonunitary dynamics 75$]$, could also be designed to further maximize the fidelity.

We then calculate the time-evolution of the realistically prepared state under the action of a linear gradient along the $y$ direction, and show the resulting density in Fig. 3(e) for a drift duration $t=10(\hbar / a F)$. After filtering out the bright-state contribution to the density, by removing the aforementioned stripes and restricting the measurement to well populated sites only, one extracts the center-of-mass motion depicted by crosses in Fig. 3(f), and recovers

the "experimental" value $C_{D}^{\exp }=1.99$. These simulations demonstrate that a dark band with $C_{D}=2$ can be loaded and probed accurately using a realistic $\Lambda$ system [Eq. [6]].

\section{Conclusion}

We proposed a realistic scheme by which flat bands with higher Chern number can be constructed through a coherent manipulation of Bloch bands. While this work focused on the simplest $\Lambda$ configuration, more bands could be involved in the construction in view of building $N$-pod settings [76, 77]; this strategy suggests a promising route to reach even higher Chern numbers $\left|C_{D}\right| \gg 2$. The dark Chern bands deriving from our scheme offer a platform where exotic fractional quantum Hall states could be explored with cold atoms, including generalized Moore-Read and Read-Rezayi states [24, 26], topological nematic states [23 and "genon" defects $23,29,78$. Interesting perspectives concern the fate of dark Chern bands in the presence of dissipation, and the coupling of Bloch bands belonging to other topological classes [79].

\section{Acknowledgements}

The authors gratefully acknowledge C. Repellin for her thoughtful comments on our manuscript, and for highlighting the possibility of realizing genons in this higher-Chern-number context. They also thank B. Irsigler for pointing out the existence of dark states and sum rules for Chern numbers in multilayer systems [62], and M. Lewenstein for triggering this collaboration.

Funding information Support of the National Science Centre (Poland) via grants 2016/23/D/ST2/00721 (M.Ł) and 2016/21/B/ST2/01086 (J.Z.) is acknowledged. N.G. is supported by the ERC Starting Grant TopoCold, and the Fonds De La Recherche Scientifique (FRS-FNRS, Belgium). This research was supported in part by PLGrid Infrastructure.

\section{A Dark state formula}

The dark state formula [Eq. (2)] for the three level $\Lambda$ system in Eq. (1) is valid in the case where $\delta=0$; no simple analytical formula for the dark state is known for the general Hamiltonian 
in Eq. (1). As moderate values of $\delta, \Delta$ are used in practice, we can use $\delta / \Omega_{1,2}$ and $\Delta / \Omega_{1,2}$ as small parameters from which a perturbative expansion can be constructed. For finite $\delta$, admixtures lead to corrections to the dark state's energy,

$$
E_{0} \approx \frac{\delta\left|\Omega_{1}\right|^{2}}{\left|\Omega_{1}\right|^{2}+\left|\Omega_{2}\right|^{2}},
$$

and to the state itself,

$$
|D(\delta)\rangle=|D(0)\rangle+\delta \frac{\Omega_{1} \Omega_{2}}{\left(\left|\Omega_{1}\right|^{2}+\left|\Omega_{2}\right|^{2}\right)^{3 / 2}}|3\rangle \ldots,
$$

and it is then referred to as the "gray" state. The above equation reveals the lowest-order correction to the dark state, which only involves the excited state $|3\rangle$. Next terms that are $O\left(\delta^{2}\right)$ and $O(\delta \Delta)$ involve all three base states $|1\rangle,|2\rangle,|3\rangle$.

Importantly, when setting $\delta=0$, the detuning $\Delta$ does not affect the $E_{0}$ eigenvalue and the state $|D(0)\rangle$ remains to be an accurate dark state. As a result, in a $\Lambda$ scheme made of Bloch bands [Fig. 1(b) in the main text], the flatness requirements on the band associated with the "third" state $\left|\psi_{3}(\mathbf{k})\right\rangle$ are substantially relaxed with respect to the other two bands.

In the standard situation where $\Omega_{1}$ and $\Omega_{2}$ couple different atomic states, the excited state $|3\rangle$ undergoes spontaneous emission. However, the spontaneous emission rate (typically in $\mathrm{MHz}$ range) dominates over single $\mathrm{kHz}$ energy scales for ultracold atom dynamics, and consequently even a small admixture to the dark state is detrimental.

In the setting considered in this work, where the excited state $|3\rangle$ is chosen as a stable optical lattice band, the admixture implied by (9) does not imply larger losses, and the Chern number is invariant with respect to perturbations of the topological dark band. This holds as long as the band gap separating dark and bright bands does not close. In fact, as seen in the following section, this requires $\delta / \Omega_{1,2} \ll 1$.

\section{B Rotating Wave Approximation}

We now consider three Bloch states with fixed quasimomentum $\mathbf{k}$ that are coupled by two time-dependent processes of frequencies $\omega_{1,2}$. We hereby discuss the validity of Eq. (1) in the main text in describing this setting.

We consider three states $|1\rangle,|2\rangle,|3\rangle$ with energies $E_{1,2,3}$. The Hamiltonian is written as

$$
H_{\Lambda, \text { lab }}=\underbrace{\hbar\left(\begin{array}{ccc}
E_{1} & 0 & 0 \\
0 & E_{2} & 0 \\
0 & 0 & E_{3}
\end{array}\right)}_{H_{a t, l a b}}+A \sin \left(\omega_{1} t\right)+B \sin \left(\omega_{2} t\right),
$$

where $A, B$ are operators that describe the couplings,

$$
A=\hbar\left(\begin{array}{ccc}
A_{11} & A_{12} & A_{13} \\
A_{12}^{*} & A_{22} & A_{23} \\
A_{13}^{*} & A_{23}^{*} & A_{33}
\end{array}\right),
$$

and similarly for $B$. We now transform to the rotating frame with the transformation $\psi=$ $U^{\dagger} \psi_{\text {lab }}$ where $U^{\dagger}=\operatorname{diag}\left[\exp \left(i E_{1} t / \hbar\right), \exp \left(i\left(E_{1}+\hbar \omega_{1}-\hbar \omega_{2}\right) t / \hbar\right), \exp \left(i\left(E_{1}+\hbar \omega_{1}\right) t / \hbar\right)\right]$. The 
transformation to the co-rotating frame is achieved by: $H_{\Lambda}=U^{\dagger} H_{\Lambda, \text { lab }} U-i \hbar U^{\dagger} \dot{U}$, where the dot denotes the time derivative. Specifically $H_{a t, r o t}$ transforms as $H_{a t, r o t}^{\prime}=\operatorname{diag}(0, \delta,-\Delta)$, where $\delta=E_{2}-E_{1}-\hbar \omega_{1}+\hbar \omega_{2}$ and $\Delta=E_{1}-E_{3}+\hbar \omega_{1}$; note that it includes the terms from the diagonal operator $-i \hbar U^{\dagger} \dot{U}$. The remaining part of the Hamiltonian transforms as $A^{\prime}=U^{\dagger} A U \sin \left(\omega_{1} t\right)$ and $B^{\prime}=U^{\dagger} B U \sin \left(\omega_{2} t\right)$, namely

$$
A^{\prime}=\frac{\hbar}{2 i}\left(\begin{array}{ccc}
-2 i A_{11} \sin \left(\omega_{1} t\right) & A_{12} e^{i \omega_{2} t}\left(e^{-2 i \omega_{1} t}-1\right) & A_{13}\left(e^{-2 i \omega_{1} t}-1\right) \\
A_{12}^{*} e^{-i \omega_{2} t}\left(1-e^{2 i \omega_{1} t}\right) & -2 i A_{22} \sin \left(\omega_{1} t\right) & -2 i A_{23} e^{-i \omega_{2} t} \sin \left(\omega_{1} t\right) \\
A_{13}^{*}\left(1-e^{2 i \omega_{1} t}\right) & -2 i A_{23}^{*} e^{i \omega_{2} t} \sin \left(\omega_{1} t\right) & -2 i A_{33} \sin \left(\omega_{1} t\right)
\end{array}\right),
$$

and analogously for $B^{\prime}$.

In the situation where all the states $|1,2,3\rangle$ belong to different spin manifolds, the Rotating Wave Approximation (RWA) directly applies and all the time-dependent, rapidly oscillating terms in the above matrix $A^{\prime}$ may be neglected. One then obtains

$$
A^{\prime} \approx \frac{\hbar}{2 i}\left(\begin{array}{ccc}
0 & 0 & -A_{13} \\
0 & 0 & 0 \\
A_{13}^{*} & 0 & 0
\end{array}\right)
$$

If two states $|1\rangle$ and $|2\rangle$ are taken within the same spin manifold, the above approximation should be replaced with

$$
A^{\prime} \approx \frac{\hbar}{2 i}\left(\begin{array}{ccc}
0 & 0 & -A_{13} \\
0 & 0 & -A_{23} e^{i t\left(\omega_{1}-\omega_{2}\right)} \\
A_{13}^{*} & A_{23}^{*} e^{-i t\left(\omega_{1}-\omega_{2}\right)} & 0
\end{array}\right) .
$$

Indeed, the time-dependent terms $\pm A_{23}^{(*)} e^{\mp i t\left(\omega_{1}-\omega_{2}\right)}$ contain the frequency $\left(\omega_{1}-\omega_{2}\right)$, which is orders of magnitude smaller then all others frequencies. In fact, it is typically of the order of $J / \hbar$, as established by the Bloch band's bandwidth. However, we point out that the approximation in Eq. (12) is still justified in this case, as long as the amplitude verifies $A_{23} \ll$ $J$; this approximation was assumed to be valid in the main text, when describing the $\Lambda$ system by Eq. (1). For instance, when $A$ is generated by a lattice modulation, the coefficients $A_{23}, A_{23}^{*}$ may be minimized by simply lowering the amplitude of the modulation. Alternatively, when different spin states are involved, $A_{23}$ can vanish due to selection rules. The justification for neglecting the terms oscillating with frequency $\left(\omega_{1}-\omega_{2}\right)$ is much more evident when the states $|1\rangle$ and $|2\rangle$ correspond to different hyperfine states; then $\hbar\left(\omega_{1}-\omega_{2}\right)$ is of the order of the hyperfine splitting, which is several orders of magnitudes larger than $J$.

The same reasoning applies to the operator $B$, with $B_{12}$ and $B_{23}$ replacing the coefficients $A_{12}$ and $A_{23}$.

\section{Dark state stability and the flatness of bands}

\section{C.1 Dark state stability}

The stability of the dark state is understood as the existence of a finite gap to the remaining two bright eigenstates in Eq. (1). Here we analyze the effects of finite $\delta$ on these gaps. We hereby drop the functional dependence on $\mathbf{k}$ from notations, as it is a conserved quantum number. 

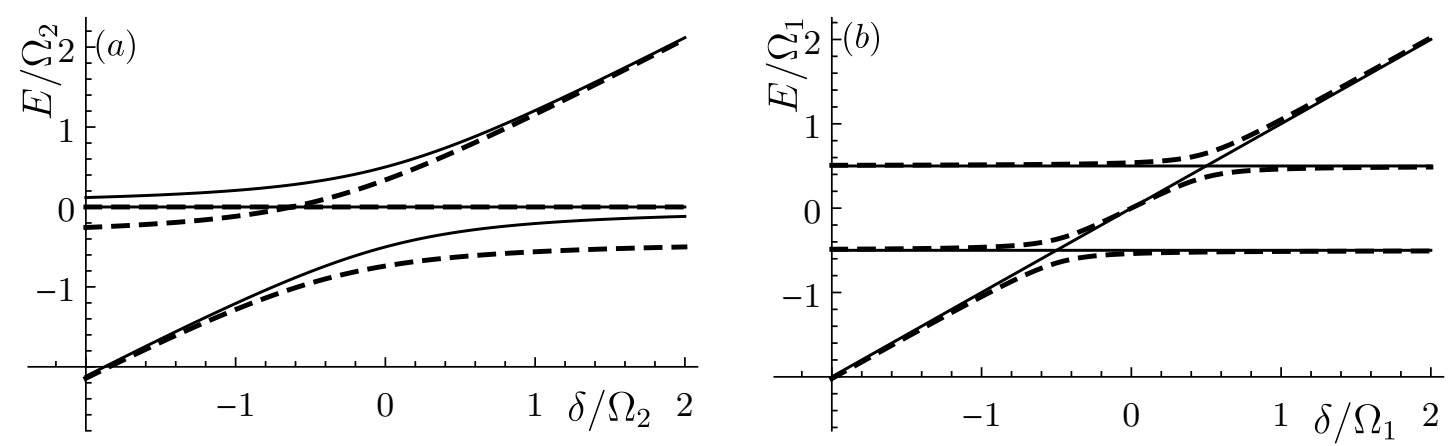

Figure 4: Three energy levels of the $H_{\Lambda}$ Hamiltonian for special values of $\Omega_{1}, \Omega_{2}$ as a function of detuning from Raman resonance condition $\delta$. Panel $(a)$ treats the case $\Omega_{1}=0, \Delta=0$ (solid lines), $\Omega_{1}=0, \Delta=0.4 \Omega_{2}$ (dashed lines). Panel (b) shows the case $\Omega_{2}=0, \Delta=0$ (solid line), $\Omega_{2}=0.4 \Omega_{1}, \Delta=0$ (dashed line).

For $\delta=0$ the bright state eigenenergies are:

$$
E_{ \pm}=\frac{1}{2}\left(-\Delta \pm \sqrt{\Delta^{2}+4 \bar{\Omega}^{2}}\right)
$$

Since the dark state energy is $E_{0}=0,\left|E_{ \pm}\right|$also gives the amplitude of the gap between bright and dark states. It is simple to check numerically that if both $\Omega_{1,2} \neq 0$, then the gap remains finite (though possibly small) for certain values of $\delta$ or $\Delta$.

When exactly one of the Rabi couplings $\left(\Omega_{1}\right.$ or $\left.\Omega_{2}\right)$ is zero, one numerically obtains that the gap may close. The other, non-zero Rabi frequency can then be chosen as the energy unit.

We first consider the case when $\Omega_{1}=0$ and $\Omega_{2} \neq 0$. Fig. 4 (a) shows the eigenvalues of $H_{\Lambda}$ as solid, thin lines in that case. For $\delta=0$, as expected, the dark state energy is precisely 0 . We see that for large $|\delta|$ the energy of one of the bright states become close to the energy of the dark/gray state, though never crosses it. The gap value is, therefore, substantially lowered which may lead to the depletion of the dark/gray state in the experiment. For $\Delta \neq 0$ the dark/gray energy level is crossed by [see Fig. [4)(a)] the bright state at a finite value of $\delta$ (dashed lines). For $\Delta \neq 0$ the crossing occurs for finite value of $\delta$. For example for $\Delta \approx 0.4 \Omega_{2}$, the crossing takes place when $\delta / \Omega_{2} \approx-0.6$, and the smaller the $|\Delta|$, the larger $\delta$ is necessary for the crossing to occur.

When $\Omega_{2}=0$ and $\Omega_{1} \neq 0$, the dark/gray state energy line is exactly linear with $\delta$ as $|D\rangle=|1\rangle$ and $|D\rangle$ is decoupled from $|2\rangle$ and $|3\rangle$. In particular, for $\delta= \pm \Omega_{2} / 2$ the gap to the bright state closes. For $\Omega_{2} \neq 0$ this energy level crossing becomes avoided [see Fig. 4(b)]. In this case the value of the detuning $\Delta$ does not change the location of the crossing, or whether it is avoided or not.

The presence of the energy level crossings (either exact or narrowly avoided) would have a detrimental effect on the construction of the dark-state band with a well-defined Chern number. However, in both cases discussed above, the crossings require large values of $\delta$ : i) $\Omega_{1}=0$ and $\delta=O\left(\Omega_{2}\right)$ or ii) $\Omega_{2}=0$ and $\delta=O\left(\Omega_{1}\right)$. Nevertheless, when the dependence of $\Omega_{1,2}$ on the quasimomentum $\mathbf{k}$ is considered, one notices that $\Omega_{1,2}$ may have zeros where $\Omega_{2,1}(\mathbf{k})$ is also small relative to its maximum value. When the values of $\Omega_{1,2}$ rapidly oscillate across the BZ, a strong upper bound on $\delta$ is therefore implied. 

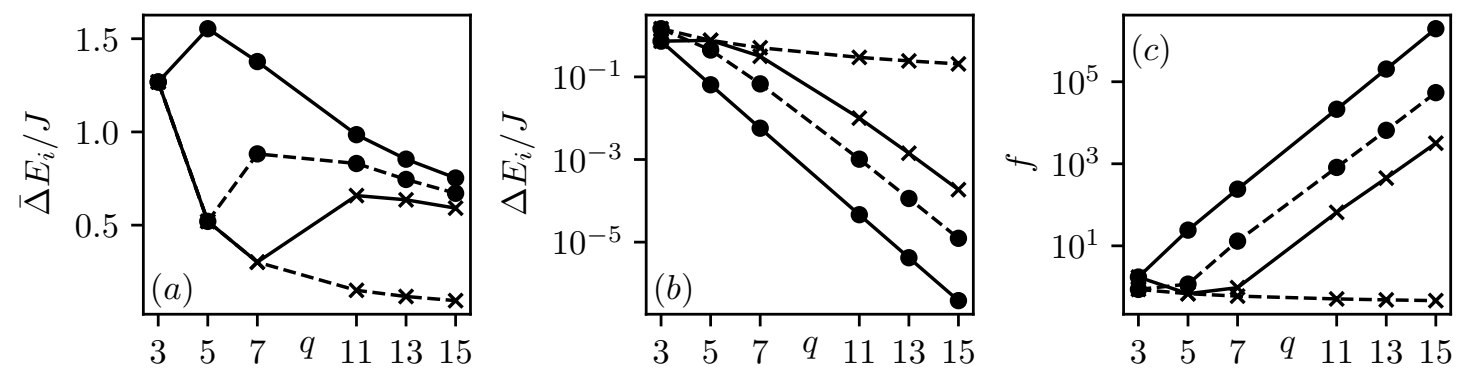

Figure 5: The panels $(a),(b)$ and $(c)$ show the properties of bands $b=1,2,3,(q-1) / 2$ of

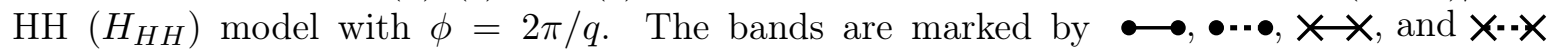
respectively. Panel $(a)$ shows the bandgap $\bar{\Delta} E_{i}$ to neighboring band, and Panel $(b)$ - the bandwidth $\Delta E_{i}$. Panel $(c)$ shows the flatness ratio $f$ for the same bands.

\section{C.2 Band flatness and Rabi frequencies variation in the Harper-Hofstadter model}

The dark state formula, Eq. (2) and Eq. (9), accurately describe the states in the dark Chern band under the following assumptions:

1. For each value of quasi-momentum $\mathbf{k}$, the detunings $\delta$ due to the finite bandwidth of the bands supporting states $|1\rangle,|2\rangle,|3\rangle$ are small compared to $\Omega_{1}, \Omega_{2}$ (as discussed in Section C).

2. The detuning $\Delta$ should not be too large, as the gap separating dark and bright bands approaches $\bar{\Omega}^{2} / 4 \Delta \rightarrow 0$ when $\Delta \gg \bar{\Omega}$.

3. States $|1\rangle,|2\rangle,|3\rangle$ are decoupled from any other states (other bands, other atomic states).

The first two requirements can be summarized in the following inequality (for each quasimomentum $\mathbf{k})$ :

$$
\delta(\mathbf{k}) \ll|\bar{\Omega}(\mathbf{k})| \ll \bar{\Delta} E_{i}, \quad i \in\{1,2,3\} .
$$

Here $\bar{\Delta} E_{i}$ is the minimum distance of the band associated with bare state $|i\rangle$ to its nearest neighbors. In the example discussed in the main text, $\bar{\Delta} E_{1}=O(J)$ simply corresponds to a gap between the lowest two Hofstadter bands; the gap $\bar{\Delta} E_{2}$ is between the top and second-top bands [in the specific choice of bands described in the main text, $\bar{\Delta} E_{2}=\bar{\Delta} E_{1}$ due to the symmetry of the spectrum; see Fig. 2(a)]; the gap $\bar{\Delta} E_{3}$ is the standard separation between $s$ and $p$ bands, in an optical lattice potential $V(x, y)=V_{x} \cos ^{2}(k x / q)+V_{y} \cos ^{2}(k y)$. The latter gap is $\bar{\Delta} E_{3}(\mathbf{k}) \approx \min \left\{\sqrt{4 E_{R} V_{y}}, \sqrt{4 E_{R} V_{x} / q^{2}}\right\}$, where $E_{R}$ is the recoil energy; sufficiently large optical-potential amplitudes $V_{x}, V_{y}$ can easily ensure sufficiently large $\bar{\Delta} E_{3}$. Altogether, only $\bar{\Delta} E_{1,2}$ give an upper bound on practical Rabi frequencies $\Omega_{1,2}(\mathbf{k})$.

It is useful to consider the ratio measuring the total variation of the Rabi frequency across the BZ:

$$
g=\min _{\mathbf{k} \in B Z}|\bar{\Omega}(\mathbf{k})| / \max _{\mathbf{k} \in B Z}|\bar{\Omega}(\mathbf{k})| .
$$

The value of this quantity provides an indication on how flat the bands associated with the states $|1\rangle$ and $|2\rangle$ have to be in view of tuning the overall amplitude of the Rabi frequencies so as to satisfy inequality (14). The flatness of the state $|3\rangle$ is not so crucial [see Appendix A]. 

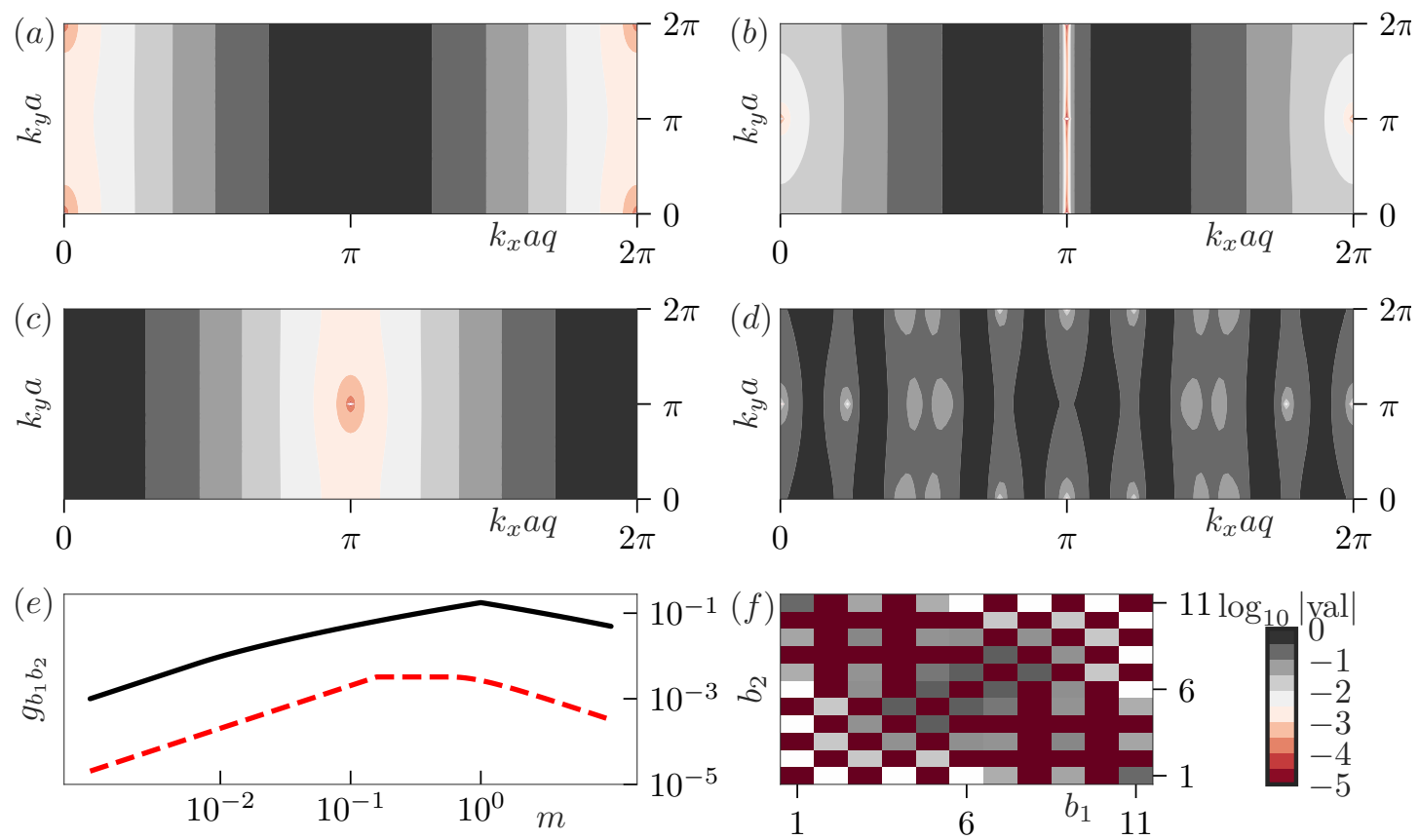

Figure 6: Panels $(a-d)$ show k-dependence across the entire BZ of $\log _{10}|\Omega|$, the Rabi frequency coupling a trivial flat $s$-band to lowest, first-excited, top, and middle band of a $\mathrm{HH}$ model, for a flux $\phi=1 / 11$. Here, $\max \left|\Omega_{s}\right|=1$ sets the normalization. Panel $(e)$ shows factor $g_{1,11}\left(g_{1,2}\right)$ as a function of $m=\max _{B Z}\left|\Omega_{1}\right|$ with solid, black (dashed red) lines. Panel $(f)$ shows $\log _{10} g_{b_{1} b_{2}}$ for $m$ maximizing its value for different $\left(b_{1}, b_{2}\right)$. Red squares correspond to $\left(b_{1}, b_{2}\right)$ where $g_{b_{1} b_{2}}=0$.

The definition of $g$ depends implicitly on the choice of the bands $b_{1,2}$; we will use the subscripted notation $g_{b_{1} b_{2}}$ below, where $b_{1}, b_{2}$ indicate the bands chosen from the Hofstadter spectrum for the states $|1\rangle,|2\rangle$ in the $\Lambda$ system.

For a particular choice of the model and bands, we consider the following flatness $f$-factor:

$$
f=\bar{\Delta} E_{i} / \Delta E_{i}
$$

where $\Delta E_{i}$ indicates the bandwidth of a given band. The inequality (14) can be satisfied when

$$
f \gg g^{-1} \text {. }
$$

The following two subsections will discuss the values of $f$ and $g$ in different cases, with an emphasis on cases where the former is maximized and the latter minimized.

\section{C.3 Band flatness - flatness factor $f$}

We consider the Hofstadter-Harper model under PBC in the thermodynamical limit [for numerics, system sizes $O(100 \times 100)$ suffice]. In Fig. $5(a),(b)$ and $(c)$ we show the bandgap $\bar{\Delta} E_{i}$, bandwidth $\Delta E_{i}$ and the flatness ratio $f$ for different $q$, for the lowest three bands and the central band (with $C=-q+1$ ) of the $\mathrm{HH}$ model. We notice that $\bar{\Delta} E_{i} / J$ is always $O(1)$, 


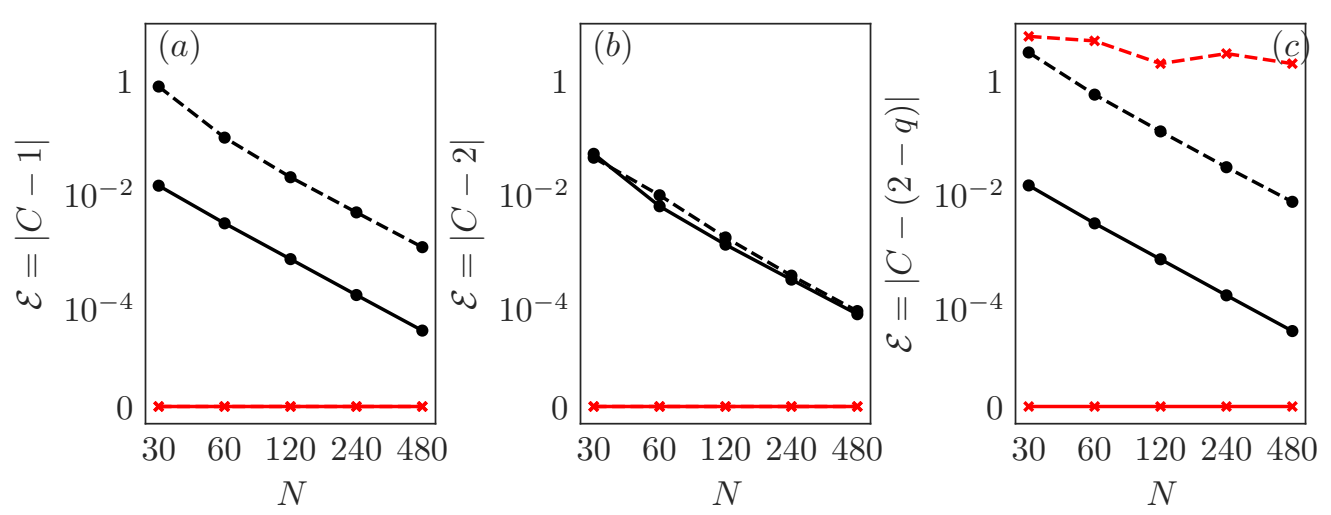

Figure 7: Approximation error $\mathcal{E}$ in Chern number computation. Panel $(a)$ shows the residual in Chern number, $C=1$ computation of lowest band of simple HH model. Panels $(b),(c)$ show same for dark state Chern number. In $(b): C_{D}=2$ for $b_{1}=1$ and $b_{2}=q$. and for panel $(c): C_{D}=-q+2$ for $b_{1}=1$ and $b_{2}=(q+1) / 2$. The curve markings are common and refer to $\bullet$, brute-force discretization method, $q=3, \bullet \cdots \bullet$ brute-force discretization method, $q=11$, $\times \times$ Fukui method, $q=3$ and $\times-\times$ Fukui method, $q=11$.

except for the band with $C \neq 1$ where it quickly drops, and that the bandwidth $\Delta E_{i} / J$ drops exponentially with $q$ already for the considered moderate values of $q$. As a result, the factor $f$ exponentially increases with $q$ for bands with $C=1$.

The behavior for $C=-q+1$ is starkly different. The bandgap decreases as $\sim 1 / q^{2}$ and the bandwidth also decreases like $\sim 1 / q$ with $q$. In the end, the maximal flatness factor $f \approx 6.5$ for the middle band is for $q=6$ and it drops down to zero as $\sim 1 / q$.

In light of this analysis, the use of the middle band (with $C \neq 1$ ), to provide states $|1\rangle$ or $|2\rangle$ in our $\Lambda$ system, is questionable.

\section{C.4 Rabi frequencies total variation - factor $g$}

Here we detail the dependence of the Rabi frequencies $\Omega_{1,2}$ on the quasimomentum $\mathbf{k}$ for the $\mathrm{HH}$ model. This aspect is important in view of satisfying Eq. (14).

First let us remark that the Rabi frequency coupling two bands with different Chern number has to be zero somewhere in the BZ. Indeed, if we assume a contrario, that $\Omega \neq 0$ for all $k_{x}, k_{y}$, we can construct the following system: $H=\delta\left|\psi_{1}\right\rangle\left\langle\psi_{1}\right|+\Omega\left(\left|\psi_{n}\right\rangle\left\langle\psi_{3}|+| \psi_{3}\right\rangle\left\langle\psi_{n}\right|\right)$ for all $k_{x}, k_{y} \in B Z$ with $\Omega \neq 0$. If the detuning $\delta$ is swept from a large positive value $\delta \gg|\Omega|$ to a large, negative value: $\delta \ll-|\Omega|$ then the eigenstate of this model changes adiabatically between $\left|\psi_{1}\right\rangle$ and $\left|\psi_{2}\right\rangle$, which would imply $C_{1}=C_{2}$, violating our assumptions. This argument says nothing about $\Omega$ connecting bands with same Chern number.

We numerically find that in the example studied in the main text, where the two bands $b_{1}$ and $b_{2}$ come from the $\mathrm{HH}$ model, and the band $b_{3}$ is a $s$ band of a (topologically trivial) 2D optical potential, $\Omega_{1}$ and $\Omega_{2}$ have coinciding zeros for roughly half of the choices of the two Hofstadter bands [see Fig. 6(f)] .

In Fig. 6(a)-(d) we plot the coupling strength $\log _{10}|\Omega|$ between the $i$-th band of the $\mathrm{HH}$ model [for $i=1,2, q,(q+1) / 2$ respectively] and a trivial flat $s$-band. We remind that the Rabi frequencies $\Omega$ are due to terms proportional to $A_{s}$ [in Eq. (6)]. The $\left|\Omega_{s}\right|$ attains a single zero 
at $\left(k_{x}, k_{y}\right)=(0,0)$ for coupling to the first band, at $\left(k_{x}, k_{y}\right)=(\pi, \pi),(\pi, 0),(0, \pi)$ for coupling to the second band, and at $\left(k_{x}, k_{y}\right)=(\pi, \pi)$ for coupling to the highest band.

The value of $g_{b_{1} b_{2}}$ does not change if both $\Omega_{1}$ and $\Omega_{2}$ are multiplied by a common factor. We assume $\max \left|\Omega_{2}\right|=1$ and $\max \left|\Omega_{1}\right|=m$. Figure $6(e)$ shows $g_{1,11}$ as a function of $m$ [compare panels $(a)$ and $(c)$ ]. For some $m=m_{*}$ the $g_{b_{1} b_{2}}$ attains the maximum value. It is also evident that the band choice can alter the factor $g_{b_{1} b_{2}}$ by several orders of magnitude. For the choice of $\left(b_{1}, b_{2}\right)=(1,11)$ for $q=11$ the maximal value of $g_{1,11}$ is $g_{1,11} \approx 0.3$.

Figure 6 $6(f)$ shows $\log _{10}\left|g_{b_{1} b_{2}}\right|$ for different choice of $b_{1}, b_{2}$ bands as a color array plot (always for $m=m_{*}$ ). We notice that for some values (indicated by deep red square) of $b_{1}, b_{2}$ (for example $b_{1}=b_{2}$ or $b_{1}=1, b_{2}=3$ ) we have $g_{b_{1} b_{2}}=0$. This is due to coinciding zeros of $\Omega_{1}(\mathbf{k})$ and $\Omega_{2}(\mathbf{k})$.

\section{Numerical evaluation of Chern numbers and the sum-rule validation}

The Chern number is defined as the integral of the Berry curvature over the BZ [Eq. (4)]. The integral can be directly computed using standard methods for numerical integration. In this work we used a regular discretization of BZ into $N \times N$ evenly-sized pieces, and integration using trapezoid prescription. When $N$ is sufficiently large, the integral for $C$ gives an error $\mathcal{E} \sim N^{-2}$ as expected. In particular, applying this method, the approximation for $C$ is not an integer number.

An alternate method has been proposed by Fukui et. al. [80]. There the integration is performed also by summation over rectangular plaquettes, but the approximant for the Chern number integral is manifestly gauge invariant, and the Chern number approximation is guaranteed to be an integer.

Figure 7 (a) shows the comparison of the two methods. There we have computed the Chern numbers of the lowest band $(C=1)$ for the standard HH model for $q \in\{3,11\}$ using bruteforce discretization and Fukui's method. In this case the method proposed by Fukui offers an obvious numerical advantage, and returns correct Chern numbers even for smallest considered discretizations. In Panel $(b)$ of the same Figure we compute the Chern number of the dark state $\left(C_{D}=2\right)$ as discussed in the main text. Panel $(c)$ shows the computation of the Chern number of a dark state band where $|1\rangle,|2\rangle$ correspond to the lowest and middle bands of the $\mathrm{HH}$ model $\left(C_{D}=-q+2\right)$. In the latter case the brute force approach converges up to the correct result much faster than the Fukui's method.

The sum rule in Eq. 5 (main text), $C_{D}=C_{1}+C_{2}-C_{3}$, has been verified to hold for all configurations of three bands $(|1\rangle,|2\rangle,|3\rangle)$ chosen among Hofstadter bands and/or a topologically trivial band; this verification was performed for the relevant cases where the Chern number of the dark-state band is well-defined (i.e. no gap closing for a particular choice of bands). We also considered the case where some of the bands stemmed from the HH model with an inverted magnetic flux: $\phi \rightarrow-\phi$. In our calculations, we considered flux values $\phi=1 / q$ with $q=3,5, \ldots, 19$.

The sum rule was also validated for different coupling operators (see Eq. 6 in the main 
text). We considered the general coupling operator

$$
\frac{1}{2} \sum_{s=1,2} A_{s} \sum_{\tilde{\mathbf{n}}=(q n, m)} f_{s, \tilde{\mathbf{n}}} \hat{a}_{\tilde{\mathbf{n}}+\tilde{\mathbf{d}}, s}^{\dagger} \hat{a}_{\tilde{\mathbf{n}}, 3}+\text { h.c. },
$$

with an arbitrary shift $\tilde{\mathbf{d}}=\left(n_{0}, 0\right)$, and also considered the case where the shift $\tilde{\mathbf{d}}$ is $s$ dependent; in the main text $\tilde{\mathbf{d}}=0$ and $f_{s, \tilde{\mathbf{n}}}=1$. We explored various forms of coupling functions $f_{s, \tilde{\mathbf{n}}}$, such as the Raman-type coupling $f_{s, \tilde{\mathbf{n}}}=\exp (i \mathbf{q} \cdot \tilde{\mathbf{n}})$, where $\mathbf{q}$ is constant. We also investigated couplings that are not localized to every $q^{\prime}$ th site, $\sum_{\tilde{\mathbf{n}}=(n, m)} f_{s, \tilde{\mathbf{n}}} \hat{a}_{\tilde{\mathbf{n}}, s}^{\dagger} \hat{a}_{\tilde{\mathbf{n}}, 3}+$ h.c., for various functions $f_{s, \tilde{\mathbf{n}}}$, including constants and Raman-type couplings $f_{s, \tilde{\mathbf{n}}}=\exp (i \mathbf{q} \cdot \tilde{\mathbf{n}})$. The sum rule was found to hold for all cases.

\section{References}

[1] M. Aidelsburger, S. Nascimbene and N. Goldman, Artificial gauge fields in materials and engineered systems, Comptes Rendus Physique 19(6), 394 (2018), doi: $10.1016 /$ j.crhy.2018.03.002.

[2] T. Ozawa, H. M. Price, A. Amo, N. Goldman, M. Hafezi, L. Lu, M. C. Rechtsman, D. Schuster, J. Simon, O. Zilberberg and I. Carusotto, Topological photonics, Rev. Mod. Phys. 91, 015006 (2019), doi:10.1103/RevModPhys.91.015006.

[3] N. Cooper, J. Dalibard and I. Spielman, Topological bands for ultracold atoms, Reviews of Modern Physics 91(1) (2019), doi:10.1103/revmodphys.91.015005.

[4] N. Goldman, J. C. Budich and P. Zoller, Topological quantum matter with ultracold gases in optical lattices, Nature Physics 12(7), 639 (2016), doi:10.1038/nphys3803.

[5] G. Jotzu, M. Messer, R. Desbuquois, M. Lebrat, T. Uehlinger, D. Greif and T. Esslinger, Experimental realization of the topological haldane model with ultracold fermions, Nature 515(7526), 237 (2014), doi:10.1038/nature13915.

[6] N. Fläschner, D. Vogel, M. Tarnowski, B. S. Rem, D.-S. Lühmann, M. Heyl, J. C. Budich, L. Mathey, K. Sengstock and C. Weitenberg, Observation of dynamical vortices after quenches in a system with topology, Nature Physics 14(3), 265 (2017), doi:10.1038/s41567017-0013-8.

[7] M. Aidelsburger, M. Atala, M. Lohse, J. T. Barreiro, B. Paredes and I. Bloch, Realization of the hofstadter hamiltonian with ultracold atoms in optical lattices, Phys. Rev. Lett. 111, 185301 (2013), doi:10.1103/PhysRevLett.111.185301.

[8] H. Miyake, G. A. Siviloglou, C. J. Kennedy, W. C. Burton and W. Ketterle, Realizing the harper hamiltonian with laser-assisted tunneling in optical lattices, Phys. Rev. Lett. 111, 185302 (2013), doi:10.1103/PhysRevLett.111.185302.

[9] M. Aidelsburger, M. Lohse, C. Schweizer, M. Atala, J. T. Barreiro, S. Nascimbène, N. R. Cooper, I. Bloch and N. Goldman, Measuring the chern number of hofstadter bands with ultracold bosonic atoms, Nature Physics 11(2), 162 (2014), doi:10.1038/nphys3171. 
[10] M. E. Tai, A. Lukin, M. Rispoli, R. Schittko, T. Menke, D. Borgnia, P. M. Preiss, F. Grusdt, A. M. Kaufman and M. Greiner, Microscopy of the interacting harper-hofstadter model in the two-body limit, Nature 546(7659), 519 (2017), doi: $10.1038 /$ nature22811.

[11] M. Lohse, C. Schweizer, O. Zilberberg, M. Aidelsburger and I. Bloch, A thouless quantum pump with ultracold bosonic atoms in an optical superlattice, Nature Physics 12(4), 350 (2015), doi:10.1038/nphys3584.

[12] S. Nakajima, T. Tomita, S. Taie, T. Ichinose, H. Ozawa, L. Wang, M. Troyer and Y. Takahashi, Topological thouless pumping of ultracold fermions, Nature Physics 12(4), 296 (2016), doi:10.1038/nphys3622.

[13] M. Lohse, C. Schweizer, H. M. Price, O. Zilberberg and I. Bloch, Exploring 4d quantum hall physics with a 2d topological charge pump, Nature 553(7686), 55 (2018), doi: $10.1038 /$ nature 25000 .

[14] T. Chalopin, T. Satoor, A. Evrard, V. Makhalov, J. Dalibard, R. Lopes and S. Nascimbene, Probing chiral edge dynamics and bulk topology of a synthetic hall system, Nature Physics (2020), doi:10.1038/s41567-020-0942-5.

[15] M. Atala, M. Aidelsburger, M. Lohse, J. T. Barreiro, B. Paredes and I. Bloch, Observation of chiral currents with ultracold atoms in bosonic ladders, Nature Physics 10(8), 588 (2014), doi:10.1038/nphys2998.

[16] M. Mancini, G. Pagano, G. Cappellini, L. Livi, M. Rider, J. Catani, C. Sias, P. Zoller, M. Inguscio, M. Dalmonte and L. Fallani, Observation of chiral edge states with neutral fermions in synthetic hall ribbons, Science 349(6255), 1510 (2015), doi:10.1126/science.aaa8736.

[17] B. K. Stuhl, H.-I. Lu, L. M. Aycock, D. Genkina and I. B. Spielman, Visualizing edge states with an atomic bose gas in the quantum hall regime, Science 349(6255), 1514 (2015), doi:10.1126/science.aaa8515.

[18] F. A. An, E. J. Meier and B. Gadway, Direct observation of chiral currents and magnetic reflection in atomic flux lattices, Science Advances 3(4), e1602685 (2017), doi $10.1126 /$ sciadv.1602685.

[19] M. Tarnowski, F. N. Ünal, N. Fläschner, B. S. Rem, A. Eckardt, K. Sengstock and C. Weitenberg, Measuring topology from dynamics by obtaining the chern number from a linking number, Nature Communications 10(1) (2019), doi:10.1038/s41467-019-09668-y.

[20] L. Asteria, D. T. Tran, T. Ozawa, M. Tarnowski, B. S. Rem, N. Fläschner, K. Sengstock, N. Goldman and C. Weitenberg, Measuring quantized circular dichroism in ultracold topological matter, Nature Physics 15(5), 449 (2019), doi:10.1038/s41567-019-0417-8.

[21] E. J. Bergholtz and Z. Liu, Topological flat band models and fractional Chern insulators, International Journal of Modern Physics B 27(24), 1330017 (2013), doi: $10.1142 /$ s021797921330017x. 
[22] T. Scaffidi and G. Möller, Adiabatic continuation of fractional chern insulators to fractional quantum hall states, Phys. Rev. Lett. 109, 246805 (2012), doi:10.1103/PhysRevLett.109.246805.

[23] M. Barkeshli and X.-L. Qi, Topological nematic states and non-abelian lattice dislocations, Phys. Rev. X 2, 031013 (2012), doi:10.1103/PhysRevX.2.031013.

[24] Z. Liu, E. J. Bergholtz, H. Fan and A. M. Läuchli, Fractional chern insulators in topological flat bands with higher chern number, Phys. Rev. Lett. 109, 186805 (2012), doi:10.1103/PhysRevLett.109.186805.

[25] Y.-F. Wang, H. Yao, C.-D. Gong and D. N. Sheng, Fractional quantum hall effect in topological flat bands with chern number two, Phys. Rev. B 86, 201101 (2012), doi:10.1103/PhysRevB.86.201101.

[26] A. Sterdyniak, C. Repellin, B. A. Bernevig and N. Regnault, Series of abelian and nonabelian states in $c>1$ fractional chern insulators, Phys. Rev. B 87, 205137 (2013), doi:10.1103/PhysRevB.87.205137.

[27] Y.-H. Wu, J. K. Jain and K. Sun, Fractional topological phases in generalized hofstadter bands with arbitrary chern numbers, Phys. Rev. B 91, 041119 (2015), doi:10.1103/PhysRevB.91.041119.

[28] G. Möller and N. R. Cooper, Fractional chern insulators in harper-hofstadter bands with higher chern number, Phys. Rev. Lett. 115, 126401 (2015), doi:10.1103/PhysRevLett.115.126401.

[29] C. Knapp, E. M. Spanton, A. F. Young, C. Nayak and M. P. Zaletel, Fractional chern insulator edges and layer-resolved lattice contacts, Phys. Rev. B 99, 081114 (2019), doi:10.1103/PhysRevB.99.081114.

[30] S. Yang, Z.-C. Gu, K. Sun and S. Das Sarma, Topological flat band models with arbitrary chern numbers, Phys. Rev. B 86, 241112 (2012), doi:10.1103/PhysRevB.86.241112.

[31] C. H. Lee, M. Claassen and R. Thomale, Band structure engineering of ideal fractional chern insulators, Phys. Rev. B 96, 165150 (2017), doi:10.1103/PhysRevB.96.165150.

[32] C. H. Lee, D. P. Arovas and R. Thomale, Band flatness optimization through complex analysis, Phys. Rev. B 93, 155155 (2016), doi:10.1103/PhysRevB.93.155155.

[33] Y.-H. Zhang, D. Mao, Y. Cao, P. Jarillo-Herrero and T. Senthil, Nearly flat chern bands in moiré superlattices, Phys. Rev. B 99, 075127 (2019), doi:10.1103/PhysRevB.99.075127.

[34] G. Chen, A. L. Sharpe, E. J. Fox, Y.-H. Zhang, S. Wang, L. Jiang, B. Lyu, H. Li, K. Watanabe, T. Taniguchi, Z. Shi, T. Senthil et al., Tunable correlated chern insulator and ferromagnetism in a moiré superlattice, Nature 579(7797), 56 (2020), doi:10.1038/s41586-020-2049-7.

[35] B. Lian, Z. Liu, Y. Zhang and J. Wang, Flat chern band from twisted bilayer mnbi $_{2} \mathrm{te}_{4}$, Phys. Rev. Lett. 124, 126402 (2020), doi:10.1103/PhysRevLett.124.126402. 
[36] E. Arimondo, Dark resonances in quantum optics, Acta Physica Polonica A 112(5), 723 (2007), doi:10.12693/aphyspola.112.723.

[37] C. Gardiner and P. Zoller, The quantum world of ultra-cold atoms and light book ii: The physics of quantum-optical devices, vol. 4, World Scientific Publishing Company, doi: $10.1142 / \mathrm{p} 983$ (2015).

[38] A. Dauphin and N. Goldman, Extracting the chern number from the dynamics of a fermi gas: Implementing a quantum hall bar for cold atoms, Physical Review Letters 111, 135302 (2013), doi:10.1103/PhysRevLett.111.135302.

[39] H. M. Price, O. Zilberberg, T. Ozawa, I. Carusotto and N. Goldman, Measurement of chern numbers through center-of-mass responses, Phys. Rev. B 93, 245113 (2016), doi:10.1103/PhysRevB.93.245113.

[40] A. Ghazaryan, T. Graß, M. J. Gullans, P. Ghaemi and M. Hafezi, Light-induced fractional quantum hall phases in graphene, Physical Review Letters 119, 247403 (2017), doi:10.1103/PhysRevLett.119.247403.

[41] J. W. McIver, B. Schulte, F.-U. Stein, T. Matsuyama, G. Jotzu, G. Meier and A. Cavalleri, Light-induced anomalous hall effect in graphene, Nature Physics 16(1), 38 (2019), doi:10.1038/s41567-019-0698-y.

[42] O. Kocharovskaya and Y. I. Khanin, Population trapping and coherent bleaching of a three-level medium by a periodic train of ultrashort pulses, Sov. Phys. JETP 63, 945 (1986).

[43] K.-J. Boller, A. Imamoğlu and S. E. Harris, Observation of electromagnetically induced transparency, Physical Review Letters 66, 2593 (1991), doi: 10.1103/PhysRevLett.66.2593.

[44] J. P. Marangos, Electromagnetically induced transparency, Journal of Modern Optics 45(3), 471 (1998), doi:10.1080/09500349808231909.

[45] L. V. Hau, S. E. Harris, Z. Dutton and C. H. Behroozi, Light speed reduction to 17 metres per second in an ultracold atomic gas, Nature 397(6720), 594 (1999), doi:10.1038/17561.

[46] A. Karawajczyk, J. Zakrzewski and W. Gawlik, Dressed-atom model of lasing without inversion in the double- $\Lambda$ configuration, Physical Review A 45, 420 (1992), doi: $10.1103 /$ PhysRevA.45.420.

[47] A. Karawajczyk and J. Zakrzewski, Semiclassical study of 'double-lambda' laser without inversion, Optics Communications 107(1-2), 145 (1994), doi:10.1016/00304018(94)90119-8.

[48] F. Vewinger, M. Heinz, R. Garcia Fernandez, N. V. Vitanov and K. Bergmann, Creation and measurement of a coherent superposition of quantum states, Phys. Rev. Lett. 91, 213001 (2003), doi:10.1103/PhysRevLett.91.213001.

[49] R. Unanyan, M. Fleischhauer, B. Shore and K. Bergmann, Robust creation and phasesensitive probing of superposition states via stimulated raman adiabatic passage (STIRAP) with degenerate dark states, Optics Communications 155(1-3), 144 (1998), doi:10.1016/s0030-4018(98)00358-7. 
[50] K. Winkler, G. Thalhammer, M. Theis, H. Ritsch, R. Grimm and J. H. Denschlag, Atommolecule dark states in a bose-einstein condensate, Phys. Rev. Lett. 95, 063202 (2005), doi: $10.1103 /$ PhysRevLett.95.063202.

[51] Y.-Y. Jau, E. Miron, A. B. Post, N. N. Kuzma and W. Happer, Push-pull optical pumping of pure superposition states, Phys. Rev. Lett. 93, 160802 (2004), doi $10.1103 /$ PhysRevLett.93.160802.

[52] M. Fleischhauer, A. Imamoglu and J. P. Marangos, Electromagnetically induced transparency: Optics in coherent media, Rev. Mod. Phys. 77, 633 (2005), doi: 10.1103/RevModPhys.77.633.

[53] G. Juzeliūnas and P. Öhberg, Slow light in degenerate fermi gases, Physical review letters 93(3), 033602 (2004).

[54] J. Ruseckas, G. Juzeliūnas, P. Öhberg and M. Fleischhauer, Non-abelian gauge potentials for ultracold atoms with degenerate dark states, Phys. Rev. Lett. 95, 010404 (2005), doi:10.1103/PhysRevLett.95.010404.

[55] G. Juzeliūnas, J. Ruseckas, P. Öhberg and M. Fleischhauer, Light-induced effective magnetic fields for ultracold atoms in planar geometries, Physical Review A 73(2), 025602 (2006).

[56] M. Łącki, M. A. Baranov, H. Pichler and P. Zoller, Nanoscale "dark state" optical potentials for cold atoms, Phys. Rev. Lett. 117, 233001 (2016), doi:10.1103/PhysRevLett.117.233001.

[57] F. Jendrzejewski, S. Eckel, T. Tiecke, G. Juzeliūnas, G. Campbell, L. Jiang and A. Gorshkov, Subwavelength-width optical tunnel junctions for ultracold atoms, Physical Review A 94(6), $063422(2016)$.

[58] Y. Wang, S. Subhankar, P. Bienias, M. Łącki, T.-C. Tsui, M. A. Baranov, A. V. Gorshkov, P. Zoller, J. V. Porto and S. L. Rolston, Dark state optical lattice with a subwavelength spatial structure, Phys. Rev. Lett. 120, 083601 (2018), doi:10.1103/PhysRevLett.120.083601.

[59] M. Lacki, P. Zoller and M. A. Baranov, Stroboscopic painting of optical potentials for atoms with subwavelength resolution, Phys. Rev. A 100, 033610 (2019), doi $10.1103 /$ PhysRevA.100.033610.

[60] D. R. Hofstadter, Energy levels and wave functions of bloch electrons in rational and irrational magnetic fields, Phys. Rev. B 14, 2239 (1976), doi:10.1103/PhysRevB.14.2239.

[61] D. J. Thouless, M. Kohmoto, M. P. Nightingale and M. den Nijs, Quantized hall conductance in a two-dimensional periodic potential, Physical Review Letters 49, 405 (1982), doi:10.1103/PhysRevLett.49.405.

[62] J. Panas, B. Irsigler, J.-H. Zheng and W. Hofstetter, Bulk topological proximity effect in multilayer systems, Phys. Rev. B 102, 075403 (2020), doi:10.1103/PhysRevB.102.075403.

[63] Y. Hatsugai, Chern number and edge states in the integer quantum hall effect, Phys. Rev. Lett. 71, 3697 (1993), doi:10.1103/PhysRevLett.71.3697. 
[64] D. Jaksch and P. Zoller, The cold atom hubbard toolbox, Annals of Physics 315(1), 52 (2005), doi:10.1016/j.aop.2004.09.010.

[65] F. Gerbier and J. Dalibard, Gauge fields for ultracold atoms in optical superlattices, New Journal of Physics 12(3), 033007 (2010), doi:10.1088/1367-2630/12/3/033007.

[66] G. Jotzu, M. Messer, F. Görg, D. Greif, R. Desbuquois and T. Esslinger, Creating statedependent lattices for ultracold fermions by magnetic gradient modulation, Phys. Rev. Lett. 115, 073002 (2015), doi:10.1103/PhysRevLett.115.073002.

[67] N. Goldman, I. Satija, P. Nikolic, A. Bermudez, M. A. Martin-Delgado, M. Lewenstein and I. B. Spielman, Realistic time-reversal invariant topological insulators with neutral atoms, Phys. Rev. Lett. 105, 255302 (2010), doi:10.1103/PhysRevLett.105.255302.

[68] C. J. Kennedy, G. A. Siviloglou, H. Miyake, W. C. Burton and W. Ketterle, Spin-orbit coupling and quantum spin Hall effect for neutral atoms without spin flips, Phys. Rev. Lett. 111, 225301 (2013), doi:10.1103/PhysRevLett.111.225301.

[69] N. Goldman, J. Beugnon and F. Gerbier, Detecting chiral edge states in the hofstadter optical lattice, Phys. Rev. Lett. 108, 255303 (2012), doi:10.1103/PhysRevLett.108.255303.

[70] X.-J. Liu, X. Liu, C. Wu and J. Sinova, Quantum anomalous hall effect with cold atoms trapped in a square lattice, Phys. Rev. A 81, 033622 (2010), doi:10.1103/PhysRevA.81.033622.

[71] A. Dauphin, D.-T. Tran, M. Lewenstein and N. Goldman, Loading ultracold gases in topological floquet bands: the fate of current and center-of-mass responses, 2D Materials 4(2), 024010 (2017), doi:10.1088/2053-1583/aa6a3b.

[72] T. Caneva, T. Calarco and S. Montangero, Chopped random-basis quantum optimization, Phys. Rev. A 84, 022326 (2011), doi:10.1103/PhysRevA.84.022326.

[73] S. J. Glaser, U. Boscain, T. Calarco, C. P. Koch, W. Köckenberger, R. Kosloff, I. Kuprov, B. Luy, S. Schirmer, T. Schulte-Herbrüggen, D. Sugny and F. K. Wilhelm, Training schrödinger's cat: quantum optimal control, The European Physical Journal D 69(12) (2015), doi:10.1140/epjd/e2015-60464-1.

[74] M. Bukov, A. G. R. Day, D. Sels, P. Weinberg, A. Polkovnikov and P. Mehta, Reinforcement learning in different phases of quantum control, Phys. Rev. X 8, 031086 (2018), doi:10.1103/PhysRevX.8.031086.

[75] S. Barbarino, J. Yu, P. Zoller and J. C. Budich, Preparing atomic topological quantum matter by adiabatic nonunitary dynamics, Phys. Rev. Lett. 124, 010401 (2020), doi: 10.1103/PhysRevLett.124.010401.

[76] C. Ding, J. Li, Z. Zhan and X. Yang, Two-dimensional atom localization via spontaneous emission in a coherently driven five-level m-type atomic system, Phys. Rev. A 83, 063834 (2011), doi:10.1103/PhysRevA.83.063834.

[77] J. Dalibard, F. Gerbier, G. Juzeliūnas and P. Öhberg, Colloquium: Artificial gauge potentials for neutral atoms, Rev. Mod. Phys. 83, 1523 (2011), doi:10.1103/RevModPhys.83.1523. 
[78] Z. Liu, G. Möller and E. J. Bergholtz, Exotic non-abelian topological defects in lattice fractional quantum hall states, Phys. Rev. Lett. 119, 106801 (2017), doi:10.1103/PhysRevLett.119.106801.

[79] M. Z. Hasan and C. L. Kane, Colloquium: Topological insulators, Rev. Mod. Phys. 82, 3045 (2010), doi:10.1103/RevModPhys.82.3045.

[80] T. Fukui, Y. Hatsugai and H. Suzuki, Chern numbers in discretized brillouin zone: Efficient method of computing (spin) hall conductances, Journal of the Physical Society of Japan 74(6), 1674 (2005), doi:10.1143/jpsj.74.1674. 